\title{
Observations of the blazar PKS 2023-07 in flaring state with H.E.S.S. and Fermi-LAT in 2016-2017 and constraints on an intrinsic cut-off
}

\section{G. Emery ${ }^{* 1}$, F. Jankowsky ${ }^{2}$, J.-P. Lenain ${ }^{1}$, J. P. Marais ${ }^{3}$, T. Mbonani ${ }^{3}$, C. Romoli ${ }^{4}$, B. van Soelen ${ }^{3}$, A. Wierzcholska ${ }^{2,5}$, M. Zacharias ${ }^{6,7}$, for the H.E.S.S. collaboration; M. Meyer ${ }^{8}$}

${ }^{1}$ Sorbonne Université, Université Paris Diderot, Sorbonne Paris Cité, CNRS/IN2P3, Laboratoire de Physique Nucléaire et de Hautes Energies, LPNHE, 4 Place Jussieu, F-75252 Paris, France

${ }^{2}$ Landessternwarte, Universität Heidelberg, Königstuhl, D69117 Heidelberg, Germany

${ }^{3}$ Department of Physics, University of the Free State, 9300 Bloemfontein, South Africa

${ }^{4}$ Max Planck Institute für Kernphysik, Saupercheckweg 1, 69117, Heidelberg, Germany

${ }^{5}$ Instytut Fizyki Jadrowej PAN, ul. Radzikowskiego 152, 31-342 Kraków, Poland

${ }^{6}$ Theoretische Physik IV, Ruhr-University Bochum, Germany

${ }^{7}$ Centre for Space Research, North-West University, Potchefstroom 2520, South Africa

${ }^{8}$ W. W. Hansen Experimental Physics Laboratory, KIPAC, Department of Physics and SLAC

National Accelerator Laboratory, Stanford University, Stanford, CA 94305, USA

E-mail: gemeryalpnhe.in2p3.fr*

\begin{abstract}
PKS 2023-07 is a flat spectrum radio quasar located at a redshift $z=1.388$, farther than any source currently detected at very high energies $(E>100 \mathrm{GeV})$. At such energies, absorption by the extragalactic background light (EBL) renders the detection of distant sources particularly challenging. The High Energy Stereoscopic System (H.E.S.S.) observed the source following reports from AGILE (April 2016) and Fermi-LAT (April 2016, September and October 2017) on high-flux states in gamma rays. During each of the three flaring periods, near-simultaneous observations were obtained with H.E.S.S., Fermi-LAT and multiple telescopes at other wavelengths. Though the source was not significantly detected by H.E.S.S., upper limits were derived for each observation period. Through constraints given by Fermi-LAT in the MeV-GeV domain and differential upper limits by H.E.S.S., we searched for an intrinsic cutoff in the EBL-corrected gamma ray spectrum of PKS 2023-07.
\end{abstract}

36th International Cosmic Ray Conference -ICRC2019-

July 24th - August 1st, 2019

Madison, Wisconsin, USA

\footnotetext{
${ }^{*}$ Speaker.

${ }^{\dagger}$ for collaboration list see PoS(ICRC2019)1177
} 


\section{Introduction}

Observations of extragalactic sources at very high energies (VHE, $E>100 \mathrm{GeV}$ ) is strongly limited by the distance of the sources in particular due to the absorption of VHE photons by the extragalactic background light (EBL), the light produced by galaxies, stars and dust during the Universe evolution [1]. In this context, active galactic nuclei (AGN) were only detected at VHE with redshifts below 1 [2] [3]. AGN are massive black holes located at the center of some galaxies which emit strongly over most of the electromagnetic spectrum while accreting matter. Around $10 \%$ of AGN power a relativistic jet. If the jet points in Earth direction, the AGN is then called a blazar and VHE photons produced by the jet can be observed.

PKS 2023-07 is a flat spectrum radio quasar (FRSQ) (sub-category of blazars [4]) located at a redshift of $z=1.388$. Even if PKS 2023-07 intrinsic emission at VHE were significant, absorption by EBL photons makes observations impossible in an average flux state. Since AGN are strongly variable, it should be possible to detect the source during an intense enough flaring activity.

The AGN Target of Opportunity (ToO) program of H.E.S.S. searches for sources in high emission state seen by other experiments at all energies (optical, X-rays, HE, VHE) and triggers observations. For AGN otherwise too faint, it can allow for detection in a reasonable amount of time. And for stronger, potentially already detected AGN, time resolved and high quality data can be obtained.

\section{High and Very High Energy Observations}

\subsection{H.E.S.S. observations}

H.E.S.S., the High Energy Stereoscopic System, is an array of telescopes located in Namibia. The array is composed of four telescopes with a mirror diameter of 12 meters called CT1 to CT4, and one with a mirror diameter of 28 meters called CT5. When a VHE photon reaches the atmosphere it interacts creating an electromagnetic shower with enough energy to produce Cherenkov light. The elliptical images obtained on the camera can be combined using the stereoscopic technique to obtain the energy and arrival direction of the VHE photon in a 5 degrees diameter field of view. H.E.S.S. can also operate in monoscopic mode using using only events detected with the CT5 telescope. In monoscopic mode, the field of view is reduced to 2.5 degrees of diameter.

H.E.S.S. observations were triggered following a high state detected with AGILE [5] and Fermi-LAT [6] in April 2016, and in September 2017 and October 2017 following private communication from the Fermi-LAT team and alerts issued with FLaapLUC [7].

The April 2016 flare was observed by H.E.S.S. for 56 minutes at the start of the flare. The observations were limited by the short observation window available from the H.E.S.S. site at the time and technical issues. During the September 2017 flare, 155 minutes of observation were possible at the start of the flare and 358 minutes at the end. The peak of the high energy flare couldn't be observed by H.E.S.S. due to technical issues. In October 2017, the decreasing phase of the high energy flare was observed for 336 minutes. Detailed observation times are displayed on Fig.1-a).

Analysis of the data taken for each flare was performed in mono mode using CT5 only. CT5 with its larger collection area possess a lower energy threshold than CT1-4 and is hence the most 


\begin{tabular}{|c|c|c|c|}
\hline Flare & Zenith & $E_{\text {threshold }}$ & Flux $\left(>E_{\text {threshold }}\right)\left(\right.$ ph.cm $\left.^{-2} . s^{-1}\right)$ \\
\hline April 2016 & 37.0 & $108 \mathrm{GeV}$ & $<3.3 \times 10^{-11}$ \\
\hline September 2017 & 20.1 & $73 \mathrm{GeV}$ & $<5.2 \times 10^{-12}$ \\
\hline October 2017 & 24.6 & $73 \mathrm{GeV}$ & $<9.1 \times 10^{-12}$ \\
\hline
\end{tabular}

Table 1: H.E.S.S. zenith of observation, energy threshold and integral upper limits

likely to detect PKS 2023-07 since absorption by the EBL is more efficient at higher energies. Each analysis was performed with two independent calibration and analysis chains to ensure robustness of the results, here the Model reconstruction [8] was used. Compatible results were obtained using the image template fitting method (ImPACT) [9]. No detection was possible for each periods and only upper limits on the flux could be derived. The 95\% C.L. differential upper limits obtained assuming a spectral index of -3 are shown on Fig.2. The 95\% C.L. integral upper limits above threshold are also produced with the same spectral hypothesis. The difference in energy threshold is due to different zenith of observation. For each flare the zenith, energy threshold and integral upper limit are compiled in Table 1.

\subsection{Fermi-LAT observations}

Fermi is a space-based gamma-ray observatory that orbits the Earth since June 2008. Its main instrument, the Large Area Telescope (LAT) sees the whole sky every two orbits (i.e. every $\sim 3 \mathrm{~h}$ ), thanks to a large field of view $(2.4 \mathrm{sr})$. Fermi-LAT is sensitive to HE photons from $\sim 20 \mathrm{MeV}$ to $>300 \mathrm{GeV}$. For most of its operation strategy, it operates in survey mode, which guarantees a complete sky survey in a sub-day time-range, while providing a fair exposure for relatively bright sources [10].

Fermi-LAT data were analysed in several subsets, in order to have contemporaneous results with the HESS observations (Table 2) for the three flares. We used the standard FermiTools ${ }^{1}$ software version 1.0.0 and the Pass 8 event selection (event class and instrument response functions P8R3_SOURCE_V2). For each flare, spectra and light curves were derived using the binned likelihood analysis gt like package in the energy range $100 \mathrm{MeV}$ to $500 \mathrm{GeV}$. The region of interest is $10^{\circ}$ of radius, and the recommended selection of time intervals are used (DATA_QUAL $>0 \& \&$ LAT_CONFIG=1). Events are selected with a zenith angle below $90^{\circ}$. The source input model was built based on the 3FGL catalogue, using the make 3FGLxml user-contributed script, and includes the Galactic interstellar emission model ( $\mathrm{gl} I_{-} i e m \_v 06$ ) and the relative isotropic diffuse emission template (iso_P8R3_SOURCE_V2). The likelihood fit is performed iteratively: first, the model is used as per the 3FGL catalogue. In a second step, sources for which $\mathrm{TS}<9$ and contributing to less than $5 \%$ of the event counts in the whole data set have all their parameters fixed. Finally, in a third iteration, the parameters of all sources beyond $3^{\circ}$ from PKS 2023-07 are fixed. Considering the short time intervals for the flares presented in this study, we assume a power-law shape for the spectrum of PKS 2023-07, even though PKS 2023-07 is best described with a log-parabola spectrum in the 3FGL catalogue $[11]^{2}$. We checked with a log-likelihood ratio test for each flare period that a log-parabolic spectral shape does not improve significantly the fit results. The most

\footnotetext{
${ }^{1}$ https://github.com/fermi-lat/Fermitools-conda

${ }^{2}$ As well as in the 4FGL catalogue [12].
} 


\begin{tabular}{|c|c|c|c|c|c|c|c|}
\hline Flare & Calendar date & MJD & MET & $\begin{array}{l}\text { duration } \\
\text { (h) }\end{array}$ & Model & $\begin{array}{l}\text { Flux } 0.1-500 \mathrm{GeV} \\
\quad\left(\text { ph.cm }{ }^{-2} \cdot s^{-1}\right)\end{array}$ & Signif \\
\hline apr16 & 12 Apr 2016 at $12: 00-14$ Apr 2016 at 12:00 & $57490.50-57492.50$ & $482155204-482328004$ & 48.0 & PL & $(2.53 \pm 0.21) \times 10^{-6}$ & 30.7 \\
\hline sept17-a & 12 Sep 2017 at $07: 00-13$ Sep 2017 at $12: 46$ & $58008.29-58009.53$ & $526892408-526999588$ & \multirow{3}{*}{108.8} & \multirow{3}{*}{ PL } & \multirow{3}{*}{$(5.77 \pm 0.87) \times 10^{-7}$} & \multirow{3}{*}{17.0} \\
\hline sept17-b & 19 Sep 2017 at $06: 33-20$ Sep 2017 at $12: 47$ & $58015.27-58016.53$ & $527495609-527604445$ & & & & \\
\hline sept17-c & 21 Sep 2017 at $06: 34-23$ Sep 2017 at $07: 24$ & $58017.27-58019.31$ & $527668446-527844258$ & & & & \\
\hline oct17 & 12 Oct 2017 at $12: 00-15$ Oct 2017 at $12: 00$ & $58038.50-58041.50$ & $529502405-529761605$ & 72.0 & PL & $(3.12 \pm 0.79) \times 10^{-7}$ & 7.9 \\
\hline
\end{tabular}

Table 2: Fermi-LAT spectral analysis

intense flare was found to happen in April 2016 but was not the most covered with H.E.S.S. The daily binned light curve is displayed on Fig.1-a).

\section{Multi-Wavelength campaign}

AGILE is a space mission containing two instruments: a gamma-ray detector, sensitive to photons with energy in the range $30 \mathrm{MeV}-50 \mathrm{GeV}$, and a hard X-ray detector, sensitive in the range $18-60 \mathrm{keV}$. AGILE reported a flux of $(2.3 \pm 0.8) \times 10^{-6} \mathrm{ph} \mathrm{cm}^{-2} \mathrm{~s}^{-1}(E>100 \mathrm{MeV})$ integrated from 2016-03-25 12:00 UT to 2016-03-27 12:00 UT.

The Neil Gehrels Swift observatory [13] is comprised of three instruments, among which the XRT [14] detects X-rays between 0.2 and $10 \mathrm{keV}$, and the UVOT observes at UV and optical wavelengths between 170 and $600 \mathrm{~nm}$. Observations were triggered following the H.E.S.S. observations. XRT data, which were all acquired in the standard photon counting mode, are analysed using the HEAS oft suite version 6.22.1. Events are cleaned using the standard criteria from xrtpipeline. Data from PKS 2023-07 are extracted within a circle of 20 pixels, and the background is taken from an annular region with an inner radius of 50 pixels and outer radius of 160 pixels. XSpec version 12.9.1p is used for the spectral analyses, where events below $0.3 \mathrm{keV}$ are excluded. Accounting for a Galactic hydrogen column of $3.24 \times 10^{20} \mathrm{~cm}^{-2}$ [15], energy flux measurements, assuming a power-law spectral shape, in the energy range $0.3-10 \mathrm{keV}$ for each exposure contemporaneous with H.E.S.S. observations are reported on Fig.1-b). Simultaneously with XRT instrument, PKS 2023-07 was monitored in the ultraviolet and optical bands with the UVOT one. All ultraviolet and optical magnitudes and fluxes have been calculated using uvot source procedure including all photons from a circular region with radius 5". The background was determined as a circular region with a radius of 10". UVOT light curves are on Fig.1-c). All data points are corrected for dust absorption.

The Automatic Telescope for Optical Monitoring (ATOM) is an optical telescope located at the H.E.S.S. site in Namibia. It provides optical monitoring on known gamma-ray emitters as well as multi-wavelength support for target-of-opportunity events and covered the 2016 flare event in $\mathrm{R}$ band. Data was reduced and analysed using ADRAS 2.4.14. Fluxes are obtained via differential photometry using between three and six custom calibrated comparison stars. The associated lightcurve is shown on Fig.1-c).

The Las Cumbres Observatory (LCO) is a worldwide network of robotic telescopes. During the 2017 flares, LCO observed the target in B,V,R,i'. Pre-reduction was performed with the LCO pipeline and differential photometry was performed using PYRAF. Differential photometry was performed using six comparison stars in the same field of view with magnitudes derived from the PanSTARR1 survey [16]. The light curve is shown on Fig.1-c). 


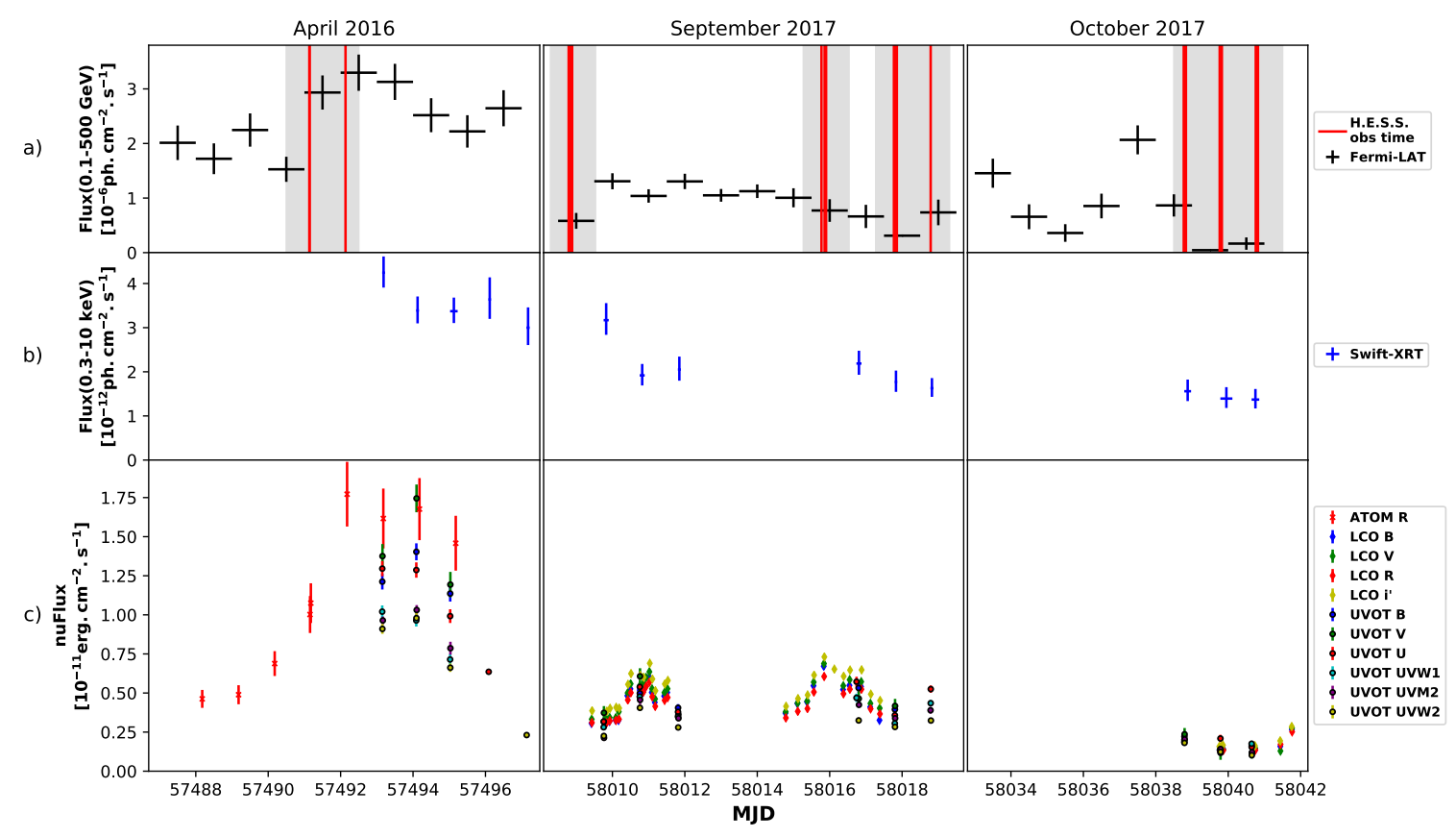

Figure 1: Multi-wavelength observations of PKS 2023-07. a) Fermi-LAT daily binned light curve integrated between $100 \mathrm{MeV}$ and $500 \mathrm{GeV}$. Red lines represent H.E.S.S. observations and the shaded grey areas cover the time windows used for the Fermi-LAT spectral analysis (shown in Fig.2). b) X-rays light curve with Swift-XRT integrated between 0.3 and $10 \mathrm{keV}$. c) optical fluxes with ATOM and LCO and optical/UV fluxes with Swift-UVOT.

\section{Discussion}

Extrapolating the Fermi-LAT power-law spectra near-simultaneous with H.E.S.S. observations for each flare (taking all Fermi-LAT observations in the grey areas on Fig.1-a), also detailed in Table 2) and taking into account absorption by the EBL with the model by Dominguez et al. [17], we can compare them with the H.E.S.S. differential upper limits (Fig.2). The 95\% C.L. upper limits at VHE probe lower fluxes than the Fermi-LAT extrapolated spectra. Clearly, a simple power-law extrapolation and standard EBL absorption are not compatible with the non-detection in April and September, whereas the H.E.S.S. upper limits for October can be explained with a simple powerlaw extrapolation of the intrinsic blazar spectrum.

To explain the non-detection by H.E.S.S., three hypothesis are tested independently. The first is the potential presence of an intrinsic cut-off in the emission region. The second is a different normalisation of the EBL than the one from the model used in the extrapolations. The last is the absorption of the VHE photons in the broad line region (BLR) around the AGN. All constraints are reported at one-sided $95 \%$ confidence levels.

To do so, we assume that the H.E.S.S. measurements are Gaussian random variables and accordingly, the H.E.S.S. upper limits in each energy bin can be translated into a Gaussian likelihood profile, see Fig.3. Then, for a given extrapolation of the Fermi-LAT SED, the predicted flux level is used in each energy band to obtain the summed likelihood. Using a profile likelihood ratio test [18], we can then evaluate if a given extrapolation is compatible with the H.E.S.S. data. 


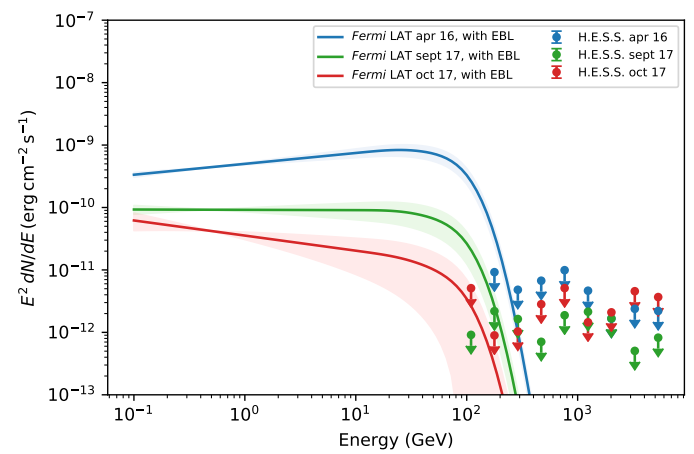

Figure 2: H.E.S.S. 95\% C.L. differential upper limits obtained for each observation campaigns. Contemporaneous Fermi-LAT power law spectra obtained from the greyed period on Fig.1-a) (see also Table 2) and extrapolated at VHE with the same power law corrected by the EBL absorption following the model by Dominguez et al. [17] are also displayed.

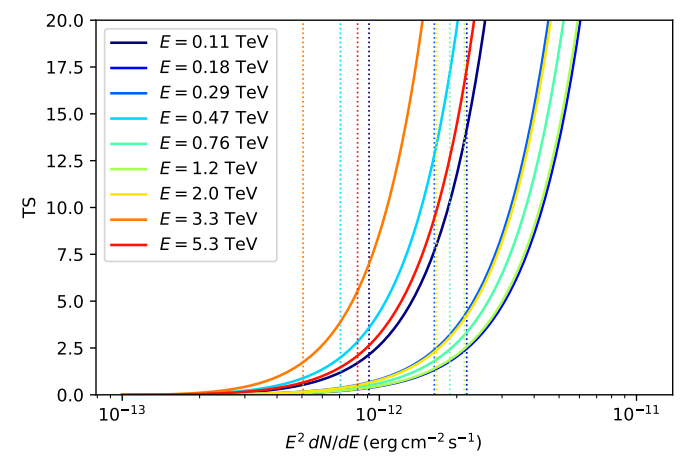

Figure 3: Test Statistic of the probability of a detection by H.E.S.S for each differential upper limit point of the September flare as a function of the flux of the source at the associated energy. The dot-lines correspond to the flux giving a detection with a probability of $95 \%$ for the associated upper limit.

First, we vary the normalization, $n_{\mathrm{EBL}}$, of the EBL photon density (modeled with model of Ref.[17]) and compute the profile likelihood as a function of $n_{\mathrm{EBL}}$. We find that one would have to increase the EBL density by at least a factor of 3.07 and 1.39 for the September 2017 and April 2016 observation campaigns, respectively. Such high levels of the EBL are not compatible with luminosity functions of galaxy and other gamma-ray measurements [17, 19].

Next, we test the hypothesis that the intrinsic spectrum is a power law modified with an exponential cut-off at energy $E_{\text {cut }}$. Again computing the profile likelihood this time as a function of $E_{\text {cut }}$ we find that $E_{\text {cut }}<128 \mathrm{GeV}$ for the April 2016 flare and $E_{\text {cut }}<35 \mathrm{GeV}$ for the September 2017 flare. In a scenario where very high-energy gamma rays are produced in inverse Compton scattering of electrons with external radiation fields, as commonly assumed for blazars [20], this would imply a cut-off or break in the electron distribution at a Lorentz factor in the comoving frame $\gamma^{\prime}=\delta_{\mathrm{D}}^{-1} \sqrt{E_{\text {cut }}(1+z) /\left(2 E_{0}\right)}$ if the electrons scatter predominantly off photons at energy $E_{0}$ in the Thompson regime [21] and where $\delta_{\mathrm{D}}$ is the Doppler factor. Assuming $\delta_{\mathrm{D}}=20$, we arrive at $\gamma^{\prime} \lesssim 3.2 \times 10^{3}$ for scattering off BLR photons at $10 \mathrm{eV}, \lesssim 1.4 \times 10^{3}$ for scattering with accretion disk photons at $50 \mathrm{eV}$, and $\lesssim 3.2 \times 10^{4}$ for scattering with photons of the dust torus at a temperature of $1000 \mathrm{~K}$ for $E_{\text {cut }}<35 \mathrm{GeV}$.

Lastly, we test if the cut-off could be due to the interaction of VHE gamma-rays with BLR photons. Following Ref.[22], we use the BLR model of Ref.[21], where we assume the ring geometry, and calculate the profile likelihood as a function of the distance $r$ of the gamma-ray emitting region to the central supermassive black hole. Searching the literature, we did not find estimates of the disk or $\mathrm{H} \beta$ luminosity and neither for black hole mass of PKS 2023-07. Therefore, we assume $L_{\text {disk }}=10^{46} \mathrm{ergs} . \mathrm{s}^{-1}$ and $L(\mathrm{H} \beta)=10^{43} \mathrm{ergs} . \mathrm{s}^{-1}$, which corresponds to a total BLR luminosity of $0.03 L_{\text {disk }}$ using the scaling relations of Ref.[21], and a black hole mass $M_{\bullet}=10^{9} M_{\odot}$, where $M_{\odot}$ is the solar mass. The H.E.S.S. data are compatible with a power-law extrapolation of the LAT spectrum and BLR absorption if the emission region is closer than $r=1.8 \times 10^{17} \mathrm{~cm} \sim 120 \mathrm{rg}_{\mathrm{g}}$ 
$\left(r=9.5 \times 10^{17} \mathrm{~cm} \sim 650 r_{g}\right)$ for the April 2016 (September 2017) flare, where $r_{g}=G M_{\bullet} / c^{2}$ is the gravitational radius with the gravitational constant $G$ and the speed of light $c$.

\section{Conclusion}

After the detection of bright activity phases at high-energy gamma rays, H.E.S.S. observed PKS 2023-07 in three occasions in April 2016, and September and October 2017 leading to no detection at very high energies. Even with the strong EBL absorption expected at a redshift of 1.388, the extrapolation of the high-energy gamma-ray spectra is incompatible with the upper limits derived for the April 2016 and September 2017 flares. A correction to the EBL density compared to the model by Dominguez et al. is ruled out due to the large correction factor which would be needed and is incompatible with the measured values. Rather, the non-detection is either caused by a break in the emitted spectrum or absorption of VHE photons in the BLR if the emission region is closer to the central black hole than several hundreds of gravitational radii.

\section{Acknowledgements}

The full H.E.S.S. acknowledgements can be found at the following link : https://www.mpi-hd.mpg.de/hfm/HESS/pages/publications/auxiliary/ HESS-Acknowledgements-2019.html

We acknowledge the use of public Fermi-LAT data and the input from Dr. Richard J. Britto and Dr. Sara Buson. We warmly thank the Neil Gehrels Swift Observatory team for the approval and prompt scheduling of ToO observations. We acknowledge the contribution of the ATOM collaboration and use of ATOM data. We acknowledge the contribution of the LCO collaboration and use of LCO data. A.W. is supported by Polish National Agency for Academic Exchange (NAWA).

\section{References}

[1] M. G. Hauser and E. Dwek, The Cosmic Infrared Background: Measurements and Implications, ARA\&A 39 (2001) 249 [astro-ph/ 0105539 ].

[2] S. Paiano, M. Landoni, R. Falomo, A. Treves, R. Scarpa and C. Righi, On the Redshift of TeV BL Lac Objects, ApJ 837 (2017) 144 [1701.04305].

[3] M. L. Ahnen, S. Ansoldi, L. A. Antonelli, P. Antoranz, C. Arcaro, A. Babic et al., Detection of very high energy gamma-ray emission from the gravitationally lensed blazar QSO B0218+357 with the MAGIC telescopes, A\&A 595 (2016) A98 [1609.01095].

[4] P. Padovani, D. M. Alexander, R. J. Assef, B. De Marco, P. Giommi, R. C. Hickox et al., Active galactic nuclei: what's in a name?, A\&A Rev. 25 (2017) 2 [1707. 07134].

[5] G. Piano, A. Bulgarelli, M. Tavani, I. Donnarumma, C. Pittori, F. Verrecchia et al., AGILE detection of a gamma-ray flare from the FSRQ PKS 2023-07, The Astronomer's Telegram 8879 (2016) .

[6] S. Ciprini and Fermi Large Area Telescope Collaboration, Fermi-LAT detection of a GeV gamma-ray flare from the blazar PKS 2023-07, The Astronomer's Telegram 8932 (2016) . 
[7] J.-P. Lenain, FLaapLUC: A pipeline for the generation of prompt alerts on transient Fermi-LAT $\gamma$-ray sources, Astronomy and Computing 22 (2018) 9 [1709.04065].

[8] M. de Naurois and L. Rolland, A high performance likelihood reconstruction of $\gamma$-rays for imaging atmospheric Cherenkov telescopes, Astroparticle Physics 32 (2009) 231 [0907 . 2610].

[9] T. Murach, M. Gajdus and R. D. Parsons, A Neural Network-Based Monoscopic Reconstruction Algorithm for H.E.S.S. II, arXiv e-prints (2015) arXiv:1509.00794 [1509.00794].

[10] W. B. Atwood, A. A. Abdo, M. Ackermann, W. Althouse, B. Anderson, M. Axelsson et al., The Large Area Telescope on the Fermi Gamma-Ray Space Telescope Mission, ApJ 697 (2009) 1071 [0902.1089].

[11] F. Acero, M. Ackermann, M. Ajello, A. Albert, W. B. Atwood, M. Axelsson et al., Fermi Large Area Telescope Third Source Catalog, ApJS 218 (2015) 23 [1501.02003].

[12] The Fermi-LAT collaboration, Fermi Large Area Telescope Fourth Source Catalog, arXiv e-prints (2019) arXiv:1902.10045 [1902.10045].

[13] N. Gehrels, G. Chincarini, P. Giommi, K. O. Mason, J. A. Nousek, A. A. Wells et al., The Swift Gamma-Ray Burst Mission, ApJ 611 (2004) 1005 [astro-ph/ 0405233 ].

[14] D. N. Burrows, J. E. Hill, J. A. Nousek, J. A. Kennea, A. Wells, J. P. Osborne et al., The Swift X-Ray Telescope, Space Sci. Rev. 120 (2005) 165 [astro-ph/ 0508071$].$

[15] P. M. W. Kalberla, W. B. Burton, D. Hartmann, E. M. Arnal, E. Bajaja, R. Morras et al., The Leiden/Argentine/Bonn (LAB) Survey of Galactic HI. Final data release of the combined LDS and IAR surveys with improved stray-radiation corrections, A\&A 440 (2005) 775 [astro-ph/ 0504140 ].

[16] K. C. Chambers, E. A. Magnier, N. Metcalfe, H. A. Flewelling, M. E. Huber, C. Z. Waters et al., The Pan-STARRS1 Surveys, arXiv e-prints (2016) arXiv:1612.05560 [1612.05560].

[17] A. Domínguez, J. R. Primack, D. J. Rosario, F. Prada, R. C. Gilmore, S. M. Faber et al., Extragalactic background light inferred from AEGIS galaxy-SED-type fractions, MNRAS 410 (2011) 2556 [1007.1459].

[18] W. A. Rolke, A. M. López and J. Conrad, Limits and confidence intervals in the presence of nuisance parameters, Nuclear Instruments and Methods in Physics Research A 551 (2005) 493 [physics/0403059].

[19] Fermi-LAT Collaboration, S. Abdollahi, M. Ackermann, M. Ajello, W. B. Atwood, L. Baldini et al., A gamma-ray determination of the Universe's star formation history, Science 362 (2018) 1031 [1812.01031].

[20] G. . Madejski and M. Sikora, Gamma-Ray Observations of Active Galactic Nuclei, ARA\&A 54 (2016) 725 .

[21] J. D. Finke, External Compton Scattering in Blazar Jets and the Location of the Gamma-Ray Emitting Region, ApJ 830 (2016) 94 [1607.03907].

[22] M. Meyer, J. D. Scargle and R. D. Blandford, Characterizing the Gamma-Ray Variability of the Brightest Flat Spectrum Radio Quasars Observed with the Fermi LAT, ApJ 877 (2019) 1 [1902.02291]. 